\title{
Low-Dose (10\%) Computed Tomography May Be Inferior to Standard-Dose CT in the Evaluation of Acute Renal Colic in the Emergency Room Setting
}

\author{
Ibraheem M. Malkawi, MD, Esther Han, DO, Christopher S. Atalla, DO, \\ Richard A. Santucci, MD, Brian O'Neil, MD, and Jason B. Wynberg, MD ${ }^{1}$
}

\begin{abstract}
Introduction: Noncontrast CT is the standard of care to evaluate nephrolithiasis. We evaluated the performance of low-dose CT (LDCT) scan for evaluation of renal colic in the emergency room (ER).

Materials and Methods: Patients visiting the ER with suspected nephrolithiasis received a standard-dose CT (SDCT) and an LDCT. Two urologists read the LDCTs and later they read SDCTs. Stone information was recorded on a diagram of the renal system. Findings on SDCTs and LDCTs were correlated through side-byside comparison of the diagrams. Later, the two urologists adjudicated all nonconcordance between SDCTs and LDCTs in an unblinded manner.

Results: Twenty-seven patients were included. SDCTs revealed 27 stones in 18 patients. Mean stone size was $3.81 \mathrm{~mm}$. LDCTs revealed 27 stones in 18 patients with a mean stone size of $4.7 \mathrm{~mm}(p=0.23)$. Overall sensitivity and specificity of LDCTs were $70 \%$ and $39 \%$, respectively. There were eight false-positive and eight false-negative stones. All the false-positive stones on LDCTs were placed in the ureter, in which all of the corresponding SDCTs were visible calcifications outside the ureter. Of the eight false-negative stones on LDCTs, seven were visible calcifications on the SDCTs and the eighth stone was $1 \mathrm{~mm}$ and was not visible. Conclusion: LDCT may not perform well in the evaluation of suspected nephrolithiasis in the acute setting. LDCT scan accurately demonstrates calcifications; however, accurate placement of calcifications in or out of the urinary tract may be diminished due to impaired resolution of soft tissue structures.
\end{abstract}

\section{Introduction}

I ONIZING RADIATION FROM medical imaging is implicated in the development of secondary malignancies. ${ }^{1-6}$ Efforts to decrease exposure to medical ionizing radiation have centered on decreasing the number of CTs ordered and using the lowest dose possible in CT scan protocols in specific scenarios. Low-dose CT (LDCT) has been studied in the setting of acute renal colic for the evaluation of suspected nephrolithiasis, ${ }^{7-10}$ including postprocessing of raw image data to reduce background noise and thereby improving image quality, ${ }^{11,12}$ with favorable results reported. We hypothesized that LDCT (10\%) is noninferior to standard-dose CT (SDCT) $(90 \%)$ in the evaluation of renal colic patients in the emergency room (ER) setting.

\section{Materials and Methods}

Institutional review board approval was obtained for this prospective study. Participation was offered to consecutive sample of patients seen in a single ER undergoing noncontrast CT scan of the abdomen and pelvis for the evaluation of renal colic. Enrolled patients first received a standard planning scout image through the Toshiba Aquilion 64 CT scanner. The scout image produced a dose plan for the CT scan in milliampere-second (mAs) units. The CT technician then performed two CT scans in immediate sequence of each other, at $90 \%$ (standard dose) and then at $10 \%$ (low dose) of the recommended dose. No postprocessing noise reduction techniques (conventional noise reduction filters, iterative reconstruction...etc.) were utilized.

\footnotetext{
${ }^{1}$ Department of Urology, Detroit Medical Center, College of Osteopathic Medicine, Michigan State University, Detroit, Michigan.

${ }^{2}$ Department of Emergency Medicine, Wayne-State University, Detroit, Michigan.

(C) Ibraheem M. Malkawi et al. 2016; Published by Mary Ann Liebert, Inc. This Open Access article is distributed under the terms of the Creative Commons Attribution Noncommercial License (http://creativecommons.org/licenses/by-nc/4.0/) which permits any noncommercial use, distribution, and reproduction in any medium, provided the original author(s) and the source are credited.
} 
FIG. 1. Low-dose scan on left shows left retroperitoneal calcification thought to be a ureteral stone, which was determined with confidence to be a phlebolith on high-dose scan (false positive).

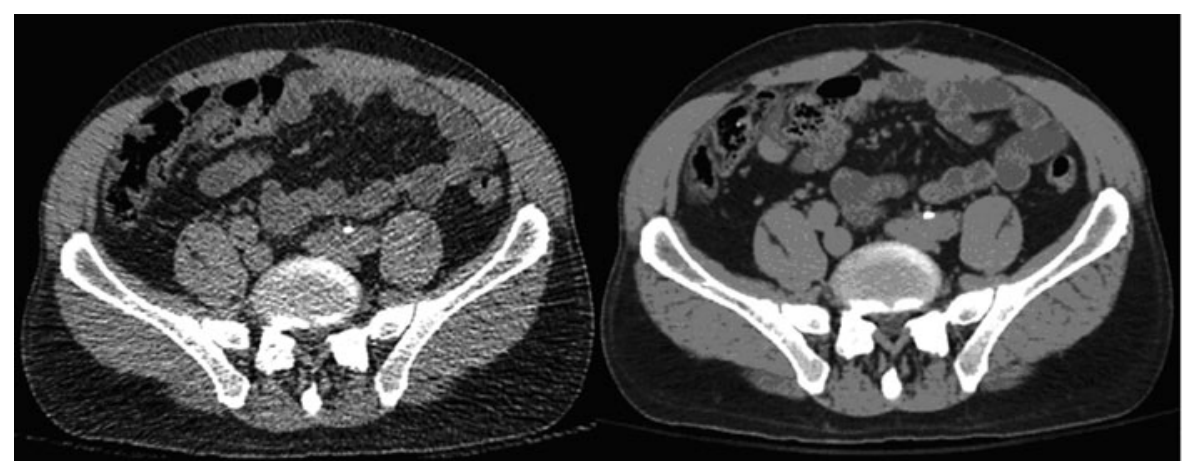

Patients did not receive a higher-than-normal dose since our CT scans were calculated at $90 \%$ and $10 \%$ of the recommended dose, accumulating to $100 \%$. The standard $(90 \%)$ dose scan was made part of the clinical chart and was reported by the radiologist, and the low-dose (10\%) scan was saved in a protected external file.

Two board-certified urologists collaboratively read the low-dose (10\%) CT scans for the presence and size of renal calculi, blinded to the radiologists reading. The stone location and size were recorded on a coronal drawing of the renal collecting system (data sheet). Four weeks later, the same two urologists read the standard-dose $(90 \%)$ films, blinded to the previous readings of the LDCT scans and to the radiology report of the SDCTs. Concordance between the two sets of CT scans was then analyzed by placing standard- and lowdose image data sheets side-by-side, with care taken to correlate individual stones based on location drawn on the data sheets. Although many consider the radiologists as the experts in interpreting radiologic studies, our study was designed to address the urologist's need to review the CT for decision-making and surgical planning.

All discrepant findings between the standard- and lowdose films were then adjudicated by the two reading urologists by viewing the standard- and low-dose films simultaneously in an unblinded manner to determine the source of the reading disparity. The clinical ER notes were reviewed for patient diagnosis and clinical plan.

A biostatistician analyzed the data using SPSS v.19.0. Continuous data were analyzed using Student's independent sample $t$-test, one-way ANOVA with a priori post hoc comparisons to evaluate pairs of differences, and MannWhitney U-tests for non-normally distributed data. Categoric data were analyzed using Fisher's exact test and the Pearson chi-square test.

\section{Results}

A total of 27 patients were evaluated from August 2011 to December 2011. Average age was 41 years (19-62), including 14 males and 13 females. The mean body mass index (BMI) was $28.7 \mathrm{~kg} / \mathrm{m}^{2}$ (range $17.7-45.9$ ) and mean recommended dose based on scout image was $301 \mathrm{mAs}$ (range 127-604). Mean dose delivered for high-dose (90\%) scans was 29.7 millisievert ( $\mathrm{mSv}$; range 8.6-51.3) and $3.5 \mathrm{mSv}$ (range 1.0-6.2) for low-dose (10\%) scans.

High-dose imaging (90\% dose) revealed 27 stones in 18 patients, with stones located in the renal parenchyma $(n=2)$, renal pelvis $(n=11)$, and ureters $(n=14)$, respectively. Eleven stones were left sided and mean stone size was $3.8 \mathrm{~mm}$ (range 1-20). Stone size by location was a mean of $2.5 \mathrm{~mm}$ in the renal parenchyma, $5 \mathrm{~mm}$ in the renal pelvis, and $3.1 \mathrm{~mm}$ in the ureter.

Low-dose imaging (10\% dose) revealed 27 stones in 18 patients, where stones were identified in the renal parenchyma $(n=2)$, renal pelvis $(n=7)$, and ureters $(n=18)$. Average reported stone size was $4.7 \mathrm{~mm}$ (range 1-20), which was not significantly different than the average stone size in the high-dose CT patients $(p=0.23)$.

When matched stone-for-stone to the high-dose scan, the overall sensitivity and specificity of low-dose (10\%) scans were $70 \%$ and $39 \%$, respectively. Age, BMI, and dose delivered did not correlate with low-dose scan accuracy ( $p=0.09, p=0.14, p=0.3$, respectively).

\section{Unblinded adjudication of reading errors}

Unblinded adjudication of the eight false-positive LDCT and eight false-negative LDCT on low-dose images was then performed.
FIG. 2. Low-dose scan on left was read as negative, when in fact a right-sided ureteral calculus was found on high-dose imaging (false negative).

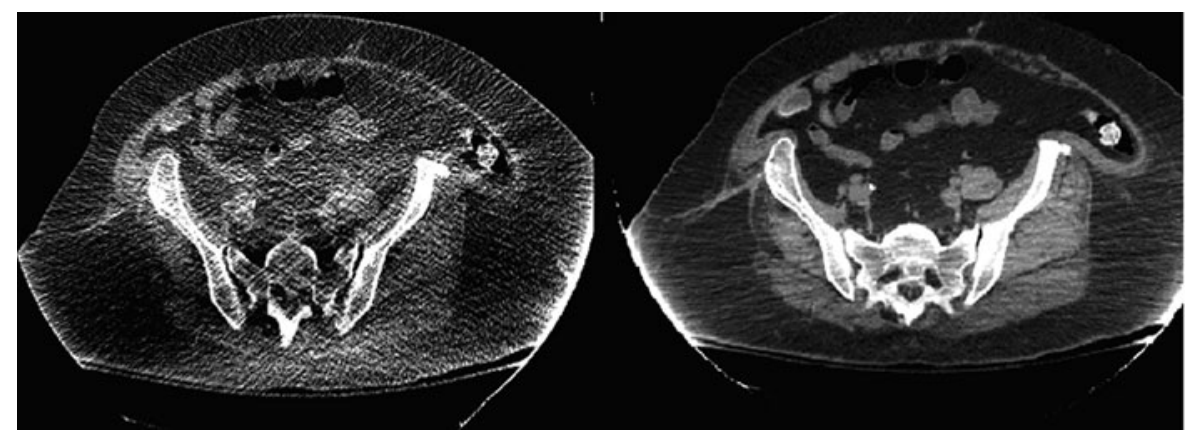




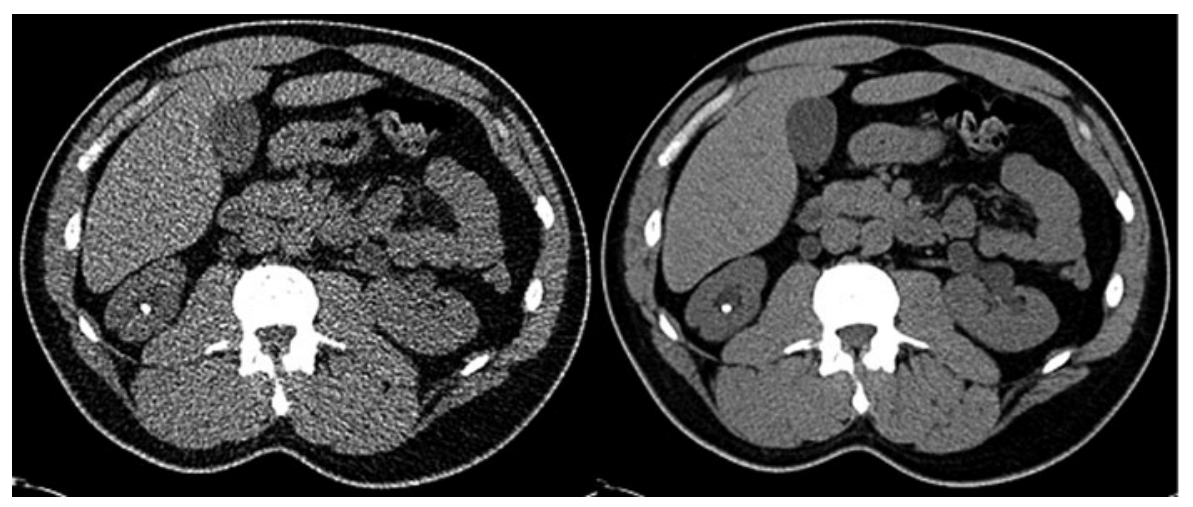

FIG. 3. Both low-dose (left) and high-dose (right) scans showed a positive finding of right renal calculus (true positive).

All of the false-positive stones $(8 / 8,100 \%)$ on LDCT were reported to be in the ureter (i.e., no false-positive findings in renal parenchyma or renal pelvis) (Fig. 1). In all the highdose films $(100 \%)$ corresponding to the false-positive, lowdose images, there were calcifications seen to be outside the ureter, for example, phlebolith at the same location of the false-positive finding on the low-dose films.

Unblinded review of the low-dose, false-negative images revealed that a calcification was in fact visible on the lowdose images at the same level as the true stone on high-dose images in $7 / 8$ (88\%) cases (four in renal pelvis, four in ureter) (Fig. 2). Neither hydronephrosis nor hydroureter was seen in any of the high-dose studies corresponding to the falsenegative, low-dose films. Only one stone was not visible on LDCT at the location of the stone on high-dose CT; the size of this stone on high-dose imaging was $1 \mathrm{~mm}$ and ipsilateral hydronephrosis was observed on LDCT.

Thus, in 15/16 (94\%) misread low-dose scans, there was accurate concordant visibility of calcifications compared with the same position on high-dose CT scan.

\section{Discussion}

In our study, the LDCT scan was inferior to SDCT scan for the evaluation of renal colic in the ER setting with an overall sensitivity and specificity of $70 \%$ and $39 \%$, respectively. The nature of our reading errors was not in fact the visibility of the calcifications - this was quite accurate (Figs. 1-3). In fact, we observed identical stone sizes between low- and high-dose CT scans. Rather, the errors we made were in accurately placing the seen calcifications on LDCT in or outside of the ureter. We believe that this placement error was due to the loss of resolution of the thin ureter on low-dose images. This problem would apply to all ureteral stones without significant ureteral dilation or without a prior regular dose CT to establish the relationship of the calcification to the ureter. We are concerned that false-positive reads may lead to unnecessary procedures. This risk may be mitigated by performing SDCT in all positive LDCT scans as part of their diagnostic evaluation. Our false-negative rate of clinically significant stones is low.

Our low sensitivity and specificity are much lower than those previously reported $(>90 \%))^{7-10}$ We believe that stone matching is a crucial part of comparing LDCT to standard high-dose CT; simply comparing the aggregate number of stones detected on LDCT compared with high-dose CT risks, overestimating performance of LDCT scans.
We do contemplate that this low-dose study may be used so long as a positive or equivocal study is followed-up with an SDCT to confirm the positive result and thus avoid any unnecessary admissions or procedures; this may need further studies to evaluate the accumulative dose reduction and the risk of missing high-risk diagnosis or complications. ${ }^{13}$

LDCT is not designed to evaluate soft tissue, however, we estimate that just as our ability to view the ureter was compromised in this study, this could potentially impair the detection of other soft tissue findings.

The presence of an alternative diagnosis (rather than nephrolithiasis) on standard high-dose imaging may be as high as $13 \%$. Poletti et al. reported the same detection rate of alternative diagnosis in their series, ${ }^{8}$ but it is more common that LDCT reports lower rates of detection for alternative diagnosis. $^{7-9,14,15}$ Nonetheless, imaging to evaluate nephrolithiasis does not require evaluation of alternate soft tissue diagnoses, for example, the use of KUB X-rays for renal stones.

We believe that low-dose (10\%) CT scan is well suited when a previous CT is available for comparison; this may be feasible for follow-up in the urology clinic or in the postoperative setting after stone removal.

\section{Limitations}

Our major limitation is the sample size, however, each patient did serve as his or her own comparator. Our study uses a prospective blinded technique, where low-dose and high-dose stones were directly matched to each other for evaluation of LDCT scans (stone matching), which makes it resistant to bias and data loss. This study was not designed to evaluate either interobserver variability or the effect of having the studies read by urologists instead of radiologists. Finally, we have not controlled for intraobserver variability, a potentially significant source of error.

\section{Conclusion}

The low-dose (90\% dose reduction) CT scan for the diagnosis of suspected nephrolithiasis in the ER setting may perform less well than previously reported. While calcifications are well seen on low-dose imaging, the loss of soft tissue resolution may result in misdiagnosis, which may lead to over or under treatment.

\section{Author Disclosure Statement}

No competing financial interests exist. 


\section{References}

1. Sountoulides P, Metaxa L, Cindolo L. Is computed tomography mandatory for the detection of residual stone fragments after percutaneous nephrolithotomy? J Endourol 2013;27:1341-1348.

2. Strauss KJ, Kaste SC. ALARA in pediatric interventional and fluoroscopic imaging: Striving to keep radiation doses as low as possible during fluoroscopy of pediatric patients-a white paper executive summary. J Am Coll Radiol 2006; 3:686-688.

3. Tack D, Sourtzis S, Delpierre I, de Maertelaer V, Gevenois PA. Low-dose unenhanced multidetector CT of patients with suspected renal colic. AJR Am J Roentgenol 2003; 180:305-311.

4. Tartari S, Rizzati R, Righi R, Deledda A, Terrani S, Benea G. Low-dose unenhanced CT protocols according to individual body size for evaluating suspected renal colic: Cumulative radiation exposures. Radiol Med 2010;115:105-114.

5. Yilmaz S, Sindel T, Arslan G, et al. Renal colic: Comparison of spiral CT, US and IVU in the detection of ureteral calculi. Eur Radiol 1998;8:212-217.

6. Lukasiewicz A, Bhargavan-Chatfield M, Coombs L, et al. Radiation dose index of renal colic protocol CT studies in the United States: A report from the American College of Radiology National Radiology Data Registry. Radiology 2014;271:445-451.

7. Moore CL, Daniels B, Ghita M, et al. Accuracy of reduceddose computed tomography for ureteral stones in emergency department patients. Ann Emerg Med 2015;65:189198.e182.

8. Poletti PA, Platon A, Rutschmann OT, Schmidlin FR, Iselin CE, Becker CD. Low-dose versus standard-dose CT protocol in patients with clinically suspected renal colic. AJR Am J Roentgenol 2007;188:927-933.

9. Kim BS, Hwang IK, Choi YW, et al. Low-dose and standard-dose unenhanced helical computed tomography for the assessment of acute renal colic: Prospective comparative study. Acta Radiol 2005;46:756-763.

10. Hamm M, Knopfle E, Wartenberg S, Wawroschek F, Weckermann D, Harzmann R. Low dose unenhanced he- lical computerized tomography for the evaluation of acute flank pain. J Urol 2002;167:1687-1691.

11. Sommer FG, Jeffrey RB, Jr., Rubin GD, et al. Detection of ureteral calculi in patients with suspected renal colic: Value of reformatted noncontrast helical CT. AJR Am J Roentgenol 1995;165:509-513.

12. Drake T, Jain N, Bryant T, Wilson I, Somani BK. Should low-dose computed tomography kidneys, ureter and bladder be the new investigation of choice in suspected renal colic?: A systematic review. Indian J Urol 2014;30:137-143.

13. Smith-Bindman R, Aubin C, Bailitz J, et al. Ultrasonography versus computed tomography for suspected nephrolithiasis. New Engl J Med 2014;371:1100-1110.

14. Pernet J, Abergel S, Parra J, et al. Prevalence of alternative diagnoses in patients with suspected uncomplicated renal colic undergoing computed tomography: A prospective study. CJEM 2015;17:67-73.

15. Cullen IM, Cafferty F, Oon SF, et al. Evaluation of suspected renal colic with noncontrast $\mathrm{CT}$ in the emergency department: A single institution study. J Endourol 2008; 22:2441-2445.

Address correspondence to: Ibraheem M. Malkawi, MD Department of Urology, DMC Harper Professional Building, Suite 1017 4160 John R Street Detroit, MI 48201

E-mail: imalkawi@dmc.org

$\begin{aligned} & \text { Abbreviations Used } \\ \mathrm{BMI} & =\text { body mass index } \\ \mathrm{CT} & =\text { computed tomography } \\ \mathrm{ER} & =\text { emergency room } \\ \mathrm{LDCT} & =\text { low-dose computed tomography } \\ \mathrm{SDCT} & =\text { standard-dose computed tomography }\end{aligned}$

